\title{
Leptin receptor expression during the progression of endometrial carcinoma is correlated with estrogen and progesterone receptors
}

\author{
Luis Fernando Méndez-López ${ }^{1}$, Angel Zavala-Pompa², Elva I. Cortés-Gutiérrez ${ }^{1}$, \\ Ricardo M. Cerda-Flores ${ }^{3}$, Martha I. Davila-Rodriguez ${ }^{1}$
}

\author{
${ }^{1}$ Division of Genetics, Biomedical Research Center Northeast, Mexican Social Security \\ Institute, Monterrey, México \\ ${ }^{2}$ Medicina Diagnóstica SA de CV, Monterrey, Mexico \\ ${ }^{3}$ School of Nursing, University of Nuevo Leon, Monterrey, México
}

Submitted: 16 February 2015

Accepted: 13 June 2015

Arch Med Sci 2017; 13, 1: 228-235

DOI: 10.5114/aoms.2017.64721

Copyright @ 2016 Termedia \& Banach

\section{Abstract}

Introduction: The hormone leptin, which is produced in the adipose tissue, may influence tumorigenesis directly via its receptor (Ob-R). Thus, a role for $\mathrm{Ob}-\mathrm{R}$ in endometrial carcinogenesis has been proposed. However, most studies neither included samples of the entire histological progression of endometrial carcinoma nor examined Ob-R jointly with the estrogen and progesterone receptors (ER and PR, respectively).

Material and methods: To determine the fluctuations of $O b-R, E R$, and $P R$ during the histological progression of endometrial carcinoma, we assessed their expression via immunohistochemistry (IHC) in six histological types of endometrium (proliferative, secretory, nonatypical and atypical hyperplasia, and endometrioid and nonendometrioid endometrial carcinoma), in which we performed histopathological and digital scoring for the quantification of receptors.

Results: We found that Ob-R expression was positively correlated with that of ER and PR $(r=1, p<0.001 ; r=0.943, p<0.005$, respectively), and there was a significant difference in Ob-R expression among proliferative normal endometrium, hyperplasias, and carcinomas, according to their relative digitally scored $\mathrm{Ob}-\mathrm{R}$ expression $(p<0.001)$. In addition, we observed that $\mathrm{Ob}-\mathrm{R}$ expression in the secretory endometrium was more similar to that of carcinomas than to its proliferative counterpart.

Conclusions: These results indicate that Ob-R expression fluctuates during endometrial carcinogenesis in correlation with ER and PR, suggesting that $O b-R$ expression in vivo is highly dependent on estrogen and progesterone activities in the endometrium and on its ER and PR status, as suggested previously by in vitro studies.

Key words: Ob-R, endometrial carcinoma, immunohistochemistry.

\section{Introduction}

Epidemiological studies have shown that obesity is associated with increased risk of several cancer types, including endometrium [1]. In Mexico, endometrial carcinoma is the fifth cause of cancer deaths in adult females and its incidence is increasing [2]. Obesity is associated with both an increased risk of developing endometrial carcinoma (it accounts for

\author{
Corresponding author: \\ Martha I. Davila-Rodriguez \\ $\mathrm{PhD}$ \\ Instituto Mexicano \\ del Seguro Social \\ 2 de abril 501 \\ Colonia Independencia \\ 64720 Monterrey, Mexico \\ Phone: 018181904036 \\ E-mail: marthadavila@ \\ cibinmty.net
}


approximately $60 \%$ of the incidence of this condition) and a worse prognosis after disease diagnosis $[1,3,4]$. Potential mechanisms linking obesity to cancer are complex and not well understood. Previous attempts to explain the effects of obesity on endometrial carcinogenesis were centered mainly on the increased levels of circulating serum estrogens and deficiency in progesterone levels. Therefore, changes in the expression of both the estrogen and progesterone receptors (ER and PR, respectively) during the histological progression of endometrial carcinoma are well documented [5].

It has been established that fat tissue is an endocrine organ that produces and secretes polypeptide hormones, adipokines, among which leptin is the most abundant [6]. Leptin is positively correlated with adipose stores and nutritional status, and important in energy balance and appetite control $[7,8]$. Research over the past few years has suggested leptin/leptin receptor (Ob-R) dysregulation to have a role in the development of a large variety of malignancies by activation of PI3K, MAPK, and STAT3 pathways [9]. Leptin induces production of inflammatory cytokines (tumor necrosis factor (TNF) $\alpha$ and interleukin (IL) 6) by macrophages, especially in obese individuals. This low-grade inflammation in individuals with metabolic syndrome in turn increases the risk of obesity-related diseases and cancer [10].

Moreover, hyperleptinemia is a common feature of obese women, who have a higher risk of endometrial cancer than women with normal weight, thus implying a direct role of the adipose tissue, via the hormone leptin [11].

Leptin through Ob-R activation has direct effects on proliferation, invasion, and production of angiogenic proteins in cancerous endometrial cells, and leptin actions are dose dependent and proportional to the expressed amount of Ob-R $[9,12,13]$.

Several studies of human endometrial carcinoma have suggested that Ob-R is downregulated in the endometrium and might be related to its differentiation status $[14,15]$, which resembles the behavior of ER and PR [16].

Furthermore, Ob-R expression has been shown to be closely related to endometrial histology (i.e., it varies according to disease or the menstrual phase) and body mass index (BMI) [17-19]. In fact, specifically, obesity increases the risk of development of a particular histological subtype, endometrioid endometrial carcinoma [20], and its precursor lesion, atypical endometrial hyperplasia [21]. However, most studies neither clearly differentiated the histological subtype of samples over the progression of endometrial carcinoma nor examined jointly Ob-R, ER, and PR expression. Taken together, the aforementioned aspects might contribute to the limitations of the current findings, such as the reported lack of differences in the expression of $\mathrm{Ob}-\mathrm{R}$ between normal and endometrial cancerous tissues [18]. Thus, the aim of this study was to assess the expression of Ob-R, ER, and PR via immunohistochemistry (IHC) in normal, hyperplasic, and endometrial cancerous tissues to increase knowledge and obtain a better prognostic value during the progression of endometrial carcinoma.

\section{Material and methods}

\section{Study population}

Paraffin-wax-embedded blocks of endometrial biopsies and clinicopathological data from 108 patients were recovered by examining files of the $\mathrm{Pa}$ thology Department located in the archives of the Mexican Institute of Social Security (IMSS), Hospitals No. 23, Gynecology and Obstetrics, and No. 25, High Specialty Medical Unit, which are both located in Monterrey, Nuevo León, Mexico, during the 2004-2006 period. Endometrial biopsies were carried out in compliance with the indications reported by physicians. The tissues were fixed in $4 \%$ formalin and embedded in paraffin wax. The mean patient age was 48 years (range: 38-71 years) and their mean BMI was $32 \mathrm{~kg} / \mathrm{m}^{2}$ (range: $\left.21-46\right)$. None of the patients had a history of exogenous hormone therapy prior to the procedure. Endometrial histology was evaluated in sections that were stained with hematoxylin and eosin ( $\mathrm{H} \& \mathrm{E})$ to confirm the initial histological diagnosis [22] and to select an adequate region for IHC. An expert pathologist assigned the menstrual cycle stage of the samples according to an idealized 28-day menstrual cycle for the normal endometrium group, which consisted of 10 biopsies of the proliferative stage and 20 biopsies of the secretory stage [22]. The hyperplasia group was divided into 19 biopsies of endometrial hyperplasia and 25 biopsies of nonatypical hyperplasia [23]. In the carcinoma group, the segregation was made according to histological type, yielding 23 biopsies of type 1 endometrioid endometrial carcinoma and 11 biopsies of type 2 nonendometrioid endometrial carcinoma [24]. Table I describes in detail the clinicopathological data of the six histological types of endometrial tissues. The Ethics Committee of the National Research Commission of the IMSS in Mexico City approved this study (registration number R-2012-785-011).

\section{Immunohistochemistry}

Sections $(4 \mu \mathrm{m})$ were cut from the blocks and placed on silanized slides (S3002; Dako North America Inc., Carpinteria, CA, USA). These were dewaxed in xylol three times, 10 min each, hydrated in a decreasing series of ethanol, and washed with distilled water. Antigen recovery was performed by heating samples to $96^{\circ} \mathrm{C}$ for 
Table I. Clinicopathological data of the six histological types of endometrial tissues

\begin{tabular}{|c|c|c|c|c|c|c|c|c|c|c|c|c|}
\hline \multirow[t]{2}{*}{$N$} & \multirow{2}{*}{$\begin{array}{c}\text { Tissue } \\
\text { Histological diagnosis }\end{array}$} & \multicolumn{2}{|c|}{ Mean \pm SD } & \multicolumn{2}{|c|}{$\begin{array}{l}\text { Menopausal } \\
\text { status }\end{array}$} & \multicolumn{3}{|c|}{$\begin{array}{l}\text { Histological } \\
\text { grade }\end{array}$} & \multicolumn{2}{|c|}{$\begin{array}{l}\text { Clinical } \\
\text { stage }\end{array}$} & \multicolumn{2}{|c|}{$\begin{array}{l}\text { Survival } \\
\text { [years] }\end{array}$} \\
\hline & & $\begin{array}{c}\text { Age } \\
\text { [years] }\end{array}$ & $\begin{array}{c}\text { BMI } \\
{\left[\mathrm{kg} / \mathrm{m}^{2}\right]}\end{array}$ & Pre & Post & 1 & 2 & 3 & Low & High & $<5$ & $>5$ \\
\hline 10 & $\begin{array}{l}\text { Proliferative } \\
\text { endometrium }\end{array}$ & $41.7 \pm 3.9$ & $27.6 \pm 2.5$ & 10 & 0 & - & - & - & - & - & - & - \\
\hline 20 & $\begin{array}{c}\text { Secretory } \\
\text { endometrium }\end{array}$ & $42.6 \pm 3.4$ & $29.8 \pm 2.9$ & 20 & 0 & - & - & - & - & - & - & - \\
\hline 25 & $\begin{array}{l}\text { Non-atypical } \\
\text { hyperplasia }\end{array}$ & $45.6 \pm 5.3$ & $31.0 \pm 4.8$ & 16 & 9 & - & - & - & - & - & - & - \\
\hline 19 & Atypical hyperplasia & $45.2 \pm 10.6$ & $31.7 \pm 5.1$ & 13 & 6 & - & - & - & - & - & - & - \\
\hline 23 & $\begin{array}{l}\text { Endometrioid } \\
\text { carcinoma }\end{array}$ & $54.8 \pm 14.8$ & $36.0 \pm 5.9$ & 7 & 16 & 8 & 12 & 3 & 19 & 4 & 12 & 11 \\
\hline 11 & $\begin{array}{c}\text { Non-endometrioid } \\
\text { carcinoma }\end{array}$ & $54.4 \pm 17.1$ & $32.2 \pm 7.1$ & 3 & 8 & 0 & 0 & 11 & 10 & 1 & 5 & 6 \\
\hline 108 & Total & $47.5 \pm 11.2$ & $31.8 \pm 5.5$ & & & & & & & & & \\
\hline
\end{tabular}

BMI - body mass index, menopausal status: pre- or postmenopausal, clinical stage: low (I-II), high (III-IV).

20 min in S1699 solution (all solutions used for IHC were from Dako North America Inc, or as specified otherwise). These were then allowed to cool at room temperature for an additional 20 min. The slides were washed with S1968 buffer for $5 \mathrm{~min}$, followed by placement in S2001 (which is an endogenous peroxidase-blocking solution) and 15 min incubation in X0909 solution, to reduce nonspecificity. Tissue sections were then incubated with the appropriate primary antibodies (anti-Ob-R sc-80255 (which covers all isoforms), anti-ER SC-56836, and anti-PR SC-810; Santa Cruz Biotechnology, Santa Cruz, CA, USA) at $1: 150,1: 300$, and $1: 100$ dilutions, respectively, in a wet chamber for $20 \mathrm{~h}$ at $4^{\circ} \mathrm{C}$. As a negative control, the primary antibody was replaced with $1 \%$ albumin in phosphate-buffered saline. A sample of breast cancer tissue that consistently exhibited a 3+ intensity after several repetitions of the experiment was taken as a positive control, to ensure the validity of the IHC procedure. The samples were incubated for $30 \mathrm{~min}$ in the $\mathrm{K0690} \mathrm{bi-}$ otinylated universal antibody, followed by a $5 \mathrm{~min}$ wash with buffer and incubation with the K0690 streptavidin-horseradish peroxidase complex for $30 \mathrm{~min}$ at room temperature. Specific reactivity was visualized by incubation with K3468 (3,3'-diaminobenzidine tetrahydrochloride (DAB)) for $10 \mathrm{~min}$, followed by immersion in $0.5 \% \mathrm{CuSO}_{4}$ for $2 \mathrm{~min}$. The slides were stained with S3309 H\&E for $1 \mathrm{~min}$, dehydrated in an ethanol series, clarified in xylol, and mounted on synthetic resin (HX787224; Merck, Darmstadt, Germany).

\section{IHC scoring}

A trained histopathologist carried out IHC scoring. Staining intensity was classified according to the following criteria: 0 , negative immunostaining; $1+$, weak staining in at least $25 \%$ of cells; $2+$, moderate expression in $25-50 \%$ of cells; and $3+$, strong expression in $\geq 50 \%$ of the cells $[25,26]$. The histopathologist assigned a histopathological score to the endometrial tissue in its entirety, to evaluate the expression of $O b-R, E R$, and $P R$ in the 108 tissues included in the study. The score corresponded to that observed in the functional glandular epithelium.

A digital score was also obtained because of its reported advantages over the histological score, i.e., it is more appropriate for the quantitative analysis of IHC with color enzyme reaction, especially those that use DAB, and it affords the most sensitive detection of antigen content [27]. All slides were visualized using an Olympus BX41 microscope (Olympus America, Inc., Melville, NY, USA) at 320× magnification, and images were captured with a Nikon D40 camera (Nikon, Inc., Melville, NY, USA). Images were digitized using the Image software (NIH ver. $1.45 \mathrm{p}$; National Institutes of Health, Bethesda, MD, USA), followed by an analysis that included the use of the rolling ball algorithm and region of interest (ROI) extraction (functional glandular epithelium); finally, we used a color deconvolution algorithm to distinguish the contributions of the DAB and H\&E stains, which allowed the independent measurement of a specific stain in areas with staining overlap by determining the amount and density of each component color channel that is required to make up the recorded color of a pixel. This algorithm reports the positive percent in a region for a given staining component in three light-transmission intensity thresholds and the average optical density of that stain. The image derived from DAB was analyzed to obtain the median optical density val- 
ue, with a range of 0-255 (black to white) [27]. A lower antigen content was associated with an optical density closer to 255 [28]. A digital score was assigned to the glandular epithelium, which was characteristic of the IHC score designated in each of the cases for the expression of Ob-R, ER, and PR in the 108 tissues included in the study.

\section{Statistical analysis}

The database was captured in the IBM SPSS statistical package (v. 22; IBM Corporation, Armonk, NY, USA). The results obtained for the three markers (Ob-R, ER, and PR) in the six histological types were processed for association in two ways: for qualitative information (histopathological score), Fisher's exact test was applied; for quantitative information (digital scoring), the Kruskal-Wallis and the Student-Newman-Keuls (SNK) tests were performed. To determine the relationship between $\mathrm{Ob}-\mathrm{R}$ and ER and PR, Spearman's correlation coefficient was applied. A $p$-value $<0.05$ was considered significant.

\section{Results}

\section{Histopathological scoring}

No statistically significant differences were found in the immunohistochemical determination of Ob-R, ER, and PR among the six endometrial tissues studied according to the histopathological scoring, as per Fisher's test. Ob-R expression was observed as cytoplasmic staining, with a $2+$ score in tissues of the proliferative endometrium, in both hyperplasia groups, and in endometrioid carcinomas, and a clear diminution to $1+$ was observed in the secretory endometrium and nonendometrioid carcinomas. ER expression exhibited a $3+$ score in the proliferative endometrium and in both hyperplasia groups, a 2+ score in endometrioid carcinomas, and a $1+$ score in the secretory endometrium and nonendometrioid carcinomas. PR expression reached a $2+$ score in the proliferative endometrium and nonatypical hyperplasia; in contrast, in atypical hyperplasias and endometrioid carcinomas, it dropped to a $1+$ score, and exhibited a negative score in the secretory endometrium and nonendometrioid carcinomas. Both ER and PR showed characteristic nuclear staining. Table II lists the immunohistochemical expression of Ob-R, ER, and PR in the six histological types of endometrial tissues included in the study, and Figure 1 includes a representative image of the IHC reactions observed for Ob-R, ER, and $P R$ in the six histological types of endometrial tissues studied here.

\section{Digital scoring}

The mean values of Ob-R expression observed in the samples studied here showed that tissues exhibited statistically significant differences in the levels of this protein and can be subgrouped in the categories according to the Kruskal-Wallis $(p<0.000)$ and SNK tests (Table II). The first group corresponded to the proliferative endometrium, which expressed the highest content of the protein. The second group corresponded to nonatypical and atypical hyperplasias, and the third group included the endometrioid carcinomas, secretory endometrium, and nonendometrioid carcinomas and expressed the lowest amount of Ob-R.

Similarly, ER expression showed statistically significant differences according to the same test (Table II); one group corresponded to the proliferative endometrium and both types of hyperplasias, and the other group, which exhibited the lowest expression level of the protein, included both types of carcinomas and the secretory endometrium.

The expression of PR was also significantly different among the samples examined. It exhibited the lowest expression level among the proteins

Table II. Immunohistochemical expression of Ob-R, ER and PR in the six histological types of endometrial tissues

\begin{tabular}{|lccccccc|}
\hline \multirow{2}{*}{$N$} & Histological diagnosis & \multicolumn{3}{c}{ Histopathological $^{\mathrm{a}}$} & \multicolumn{3}{c|}{ Digital score (median \pm SD) $^{\mathrm{b}}$} \\
\cline { 3 - 8 } & & Ob-R & ER & PR & Ob-R & ER $^{* *}$ & PR $^{* * *}$ \\
\hline 10 & Proliferative endometrium (PE) & $(++)$ & $(+++)$ & $(++)$ & $26 \pm 9$ & $30 \pm 13$ & $113 \pm 45$ \\
\hline 20 & Secretory endometrium (SE) & $(+)$ & $(++)$ & $(-)$ & $143 \pm 29$ & $153 \pm 56$ & $208 \pm 44$ \\
\hline 25 & Non-atypical hyperplasia (NAH) & $(++)$ & $(+++)$ & $(++)$ & $87 \pm 35$ & $33 \pm 21$ & $162 \pm 67$ \\
\hline 19 & Atypical hyperplasia (AH) & $(++)$ & $(+++)$ & $(+)$ & $98 \pm 34$ & $42 \pm 22$ & $161 \pm 59$ \\
\hline 23 & Endometrioid carcinoma (EEC) & $(++)$ & $(++)$ & $(+)$ & $141 \pm 64$ & $132 \pm 81$ & $205 \pm 47$ \\
\hline 11 & Non-endometrioid carcinoma (NEC) & $(+)$ & $(+)$ & $(-)$ & $177 \pm 68$ & $214 \pm 37$ & $224 \pm 41$ \\
\hline Kruskal-Wallis test & & & & $p<0.001$ & $p<0.001$ & $p<0.001$ \\
\hline
\end{tabular}

${ }^{a}$ Histopathological score is shown as average per group; ${ }^{b}$ Digital score is shown as the optical median value of groups $\pm S D$. SNK test: ${ }^{*} P E \neq S E \neq N A H=A H \neq E E C=N E C ;{ }^{* *} P E \neq S E \neq N A H=A H \neq E E C \neq N E C ;{ }^{* * *} P E \neq S E \neq N A H=A H \neq E E C=N E C$. Spearman correlation: $O b-R$ vs. $E R(r=1, p<0.0001), O b-R$ vs. $P R(r=0.943, p<0.005)$. Ob-R-leptin receptor, ER-estrogen receptor, $P R$ - progesterone receptor. 
$\mathrm{Ob}-\mathrm{R}$

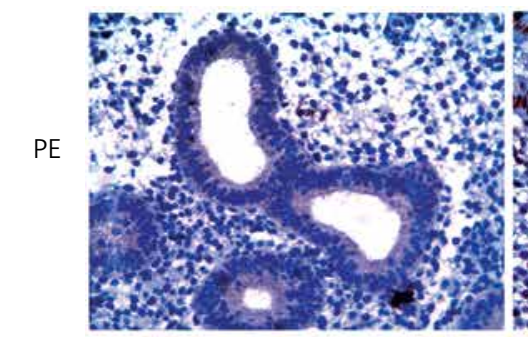

$\mathrm{NAH}$

$\mathrm{AH}$

EEC
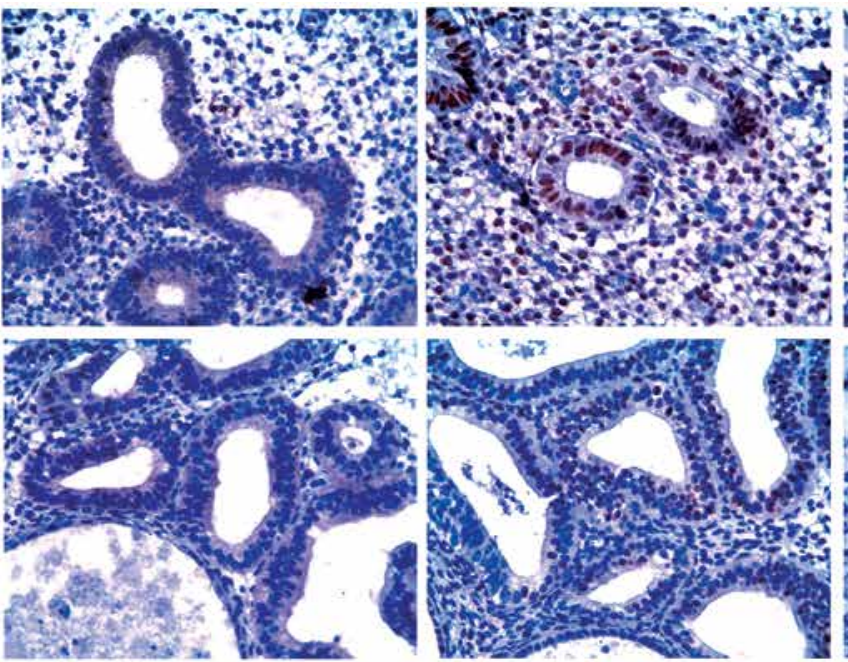

$\lim ^{2}$
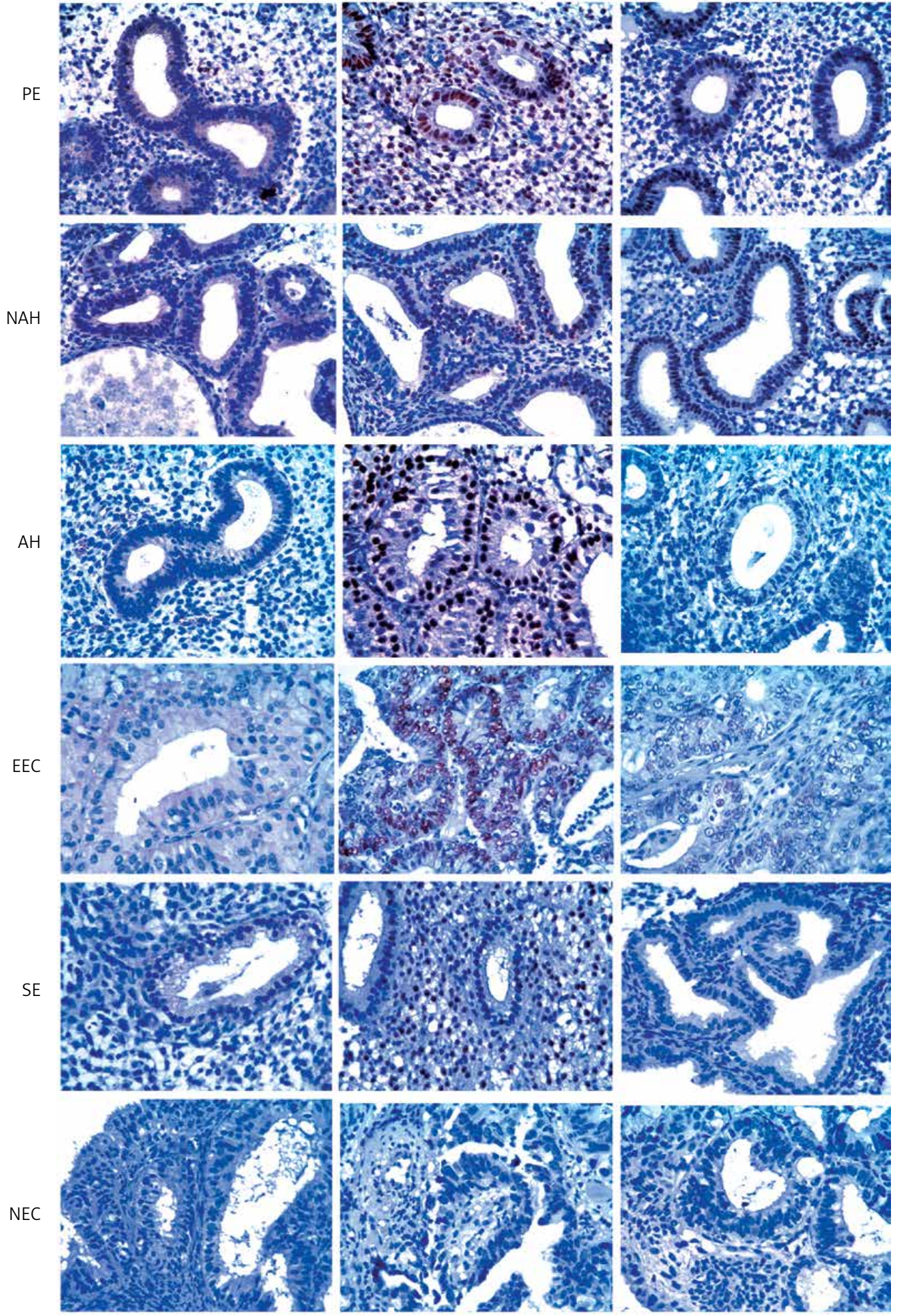

$0.1 \mathrm{~mm}$

Figure 1. Representative examples of IHC signals for Ob-R, ER, and PR in the six histological types of endometrial tissues studied here. For illustrative purposes, the tissues were ordered according to their relative expression score (high to low score for Ob-R)

$O b-R$ - leptin receptor, $E R$ - estrogen receptor, $P R$ - progesterone receptor, $P E$ - proliferative endometrium, NAH - nonatypical hyperplasia, AH - atypical hyperplasia, EEC - endometrioid carcinoma, SE - secretory endometrium, NEC - nonendometrioid endometrial carcinoma. 
analyzed here, and was subgrouped in the same manner as that described for Ob-R (Table II) (proliferative endometrium, nonatypical and atypical hyperplasia, endometrioid carcinomas, proliferative endometrium, and nonendometrioid carcinomas).

Finally, Spearman's correlation indicated that Ob-R expression was positively correlated with the expression of both ER $(r=1, p<0.0001)$ and PR $(r=0.943, p=0.005)$.

\section{Discussion}

Increased serum leptin levels is a common feature of obese women at risk of developing endometrial carcinoma [11]. Leptin effects on proliferation and invasion in cancerous endometrial cells are dose dependent and proportional to the expressed amount of Ob-R $[9,12,13]$. Thus its behavior during the progression of endometrial carcinoma might be related to the histological features of tissues.

We found that the leptin receptor was downregulated during the histological progression of endometrial carcinoma, and that its expression was correlated with both the estrogen and progesterone receptors. We also found that Ob-R expression was clearly opposite between the two normal endometrial phases included in the study: the proliferative and secretory endometrium. As reported previously, we found that Ob-R was downregulated in parallel with endometrial dedifferentiation (data not shown) and was diminished in tumor-tissue zones compared with histologically normal tissue zones of the same patient [14]. We also found that endometrial tumors with a solid appearance (higher histological grade) tended to have lower Ob-R expression compared with those that were well differentiated [15]. Moreover, we provide evidence that both types of endometrial cancer lost Ob-R expression markedly compared with the proliferative endometrium, which was in contrast with a previous report of a lack of significant differences between normal proliferative tissues and cancerous tissues of the endometrium [18].

Furthermore, we found that Ob-R expression in hyperplasias was among cancerous and proliferative tissues. This was in line with a model of Ob-R downregulation during the progression of endometrial carcinoma because these lesions are considered the precursors of endometrioid carcinoma [29].

In addition, we showed that the normal proliferative and secretory phases of the endometrium exhibited contrasting levels of $\mathrm{Ob}-\mathrm{R}$, with the highest expression observed in the proliferative phase, and a marked reduction in the secretory phase. Furthermore, we found that Ob-R expression in the proliferative phase was associated to a greater extent with hyperplasias compared with the secre- tory phase. Similarly, the secretory phase exhibited a greater equivalence with carcinomas than it did with the proliferative phase, which clarified its status between the normal endometrial phases. This result was in contrast with the reported maximal expression of Ob-R RNA levels in the secretory stage [17]; however, RNA expression does not necessarily match protein levels [30], which might have been the case here. Otherwise, the low levels of the Ob-R protein observed in the secretory phase were consistent with the reported effects of progesterone on its downregulation [31].

The reported levels of $O b-R$ are in line with the experimental evidence of the mitogenic effects of leptin [9], given that the higher levels of Ob-R were found in proliferative and hyperplasic tissues. In contrast, in cancerous tissues Ob-R expression is downregulated, which is in agreement with the progression toward the independence of hormones in endometrial tumors [32].

Furthermore, we report for the first time a correlation between $O b-R$ and ER and PR during endometrial carcinogenesis. This implies that $\mathrm{Ob}-\mathrm{R}$ expression is regulated by those hormones. Although this phenomenon is not understood completely, fluctuations in Ob-R may be caused by positive dose-dependent regulation of estrogens by the expression of leptin and $\mathrm{Ob}-\mathrm{R}$ through direct ER activation [33], whereas the regulation of progesterone through the activation of PR diminishes its expression [31]. This might explain the high Ob-R levels observed in hyperplasias and proliferative endometrium because both are characterized by conditions of high estrogen and low progesterone levels, including a loss of PR expression in hyperplasias [3]. In addition, those tissues also possess high leptin levels. However, in contrast with the downregulation of $\mathrm{Ob}-\mathrm{R}$ in the normal secretory phase in patients with hyperplasias, hyperleptinemia may account for an even greater hormonal misbalance and enhance carcinogenesis in obese patients.

These results are supported by the effects of hyperleptinemia in normal follicular development and ovulation, including the disruption of normal menstrual cycles [34]. Moreover, women diagnosed with atypical hyperplasia have a 10 times greater probability of developing carcinomas [35].

The results obtained here for ER and PR reaffirm previous studies in which the IHC signals of these proteins were diminished in carcinomas compared with normal endometrial tissues and hyperplasias. Moreover, the best-differentiated carcinomas exhibited a greater expression level of both receptors compared with poorly differentiated tumors [36].

The limitations of the present study must be considered when interpreting its results. First, we used a digital scoring approach for the analysis of DAB staining because of the reported advantages 
of this method, which enhanced the chances of capturing subtle changes in antigen content [27]. Second, the antibody used for Ob-R detection reacts with all isoforms of the protein, whereas the effects of leptin are restricted to the Ob-Rb isoform - the only one that possesses signal-transducing capabilities [37]. Finally, we did not assess serum hormone levels in the patients included in the present study. Despite these limitations, we clarified the pattern of expression of the Ob-R protein during the histological progression of endometrial carcinoma, which had not been reported previously. We clearly differentiated the histological subtypes of samples over tumorigenesis: control groups, proliferative and secretory endometrium, atypical and nonatypical hyperplasia, and endometrial carcinoma types I and II. We reported for the first time the correlation between $\mathrm{Ob}-\mathrm{R}$ and the receptors of the steroid hormones estrogen and progesterone, which are currently the most valuable prognostic biomarkers of this neoplasia [38].

In conclusion, during the progression of endometrial carcinoma, Ob-R was downregulated and was correlated with ER and PR expression. However, its overexpression in hyperplasias might enhance its susceptibility to malignancy in patients with obesity because of hyperleptinemia. Moreover, although Ob-R expression appeared to be regulated by estrogen and progesterone, during carcinogenesis, the expression of the three markers was lost altogether after the differentiation of endometrial tissues. The analysis of Ob-R together with hormone receptors suggests that the assessment of these receptors in endometrial carcinoma has a better prognostic value than does the analysis of Ob-R and hormone receptors alone.

\section{Acknowledgments}

This study was supported by Fondo de Investigación en Salud, Instituto Mexicano del Seguro Social (FIS/IMSS/PROT/G12/1144).

\section{Conflict of interest}

The authors declare no conflict of interest.

\section{References}

1. Calle EE, Kaaks R. Overweight, obesity and cancer: epidemiological evidence and proposed mechanisms. Nat Rev Cancer 2004; 4: 579-91.

2. Salazar-Martinez E, Lazcano-Ponce EC, Gonzalez LiraLira G, Escudero-De los Rios P, Salmeron-Castro J, Hernandez-Avila M. Reproductive factors of ovarian and endometrial cancer risk in a high fertility population in Mexico. Cancer Res 1999; 59: 3658-62.

3. Kaaks R, Lukanova A, Kurzer MS. Obesity, endogenous hormones, and endometrial cancer risk: a synthetic review. Cancer Epidemiol Biomarkers Prev 2002; 11: 1531-43.

4. Gottwald L, Dukowicz A, Piekarski J, et al. Isolated metastasis to the foot as an extremely rare presenting feature of primary endometrial cancer. Arch Med Sci 2012; 8: 172-4.

5. Lessey BA, Killam AP, Metzger DA, Haney AF, Greene GL, McCarty KS Jr. Immunohistochemical analysis of human uterine estrogen and progesterone receptors throughout the menstrual cycle. J Clin Endocrinol Metab 1988; 67: 334-40.

6. Stępień M, Stępień A, Banach M, et al. New obesity indices and adipokines in normotensive patients and patients with hypertension comparative pilot analysis. Angiology 2014; 65: 333-42.

7. Huang L, Li C. Leptin: a multifunctional hormone. Cell Res 2000; 10: 81-92.

8. Stępień M, Wlazeł RN, Paradowski M, et al. Serum concentrations of adiponectin, leptin, resistin, ghrelin and insulin and their association with obesity indices in obese normo-and hypertensive patients - pilot study. Arch Med Sci 2012; 8: 431-6.

9. Gao J, Tian J, Lv Y, et al. Leptin induces functional activation of cyclooxygenase-2 through JAK2/STAT3, MAPK/ ERK, and PI3K/AKT pathways in human endometrial cancer cells. Cancer Sci 2009; 100: 389-95.

10. Martin-Romero C, Santos-Alvarez J, Goberna R, Sanchez-Margalet V. Human leptin enhances activation and proliferation of human circulating T lymphocytes. Cell Immunol 2000; 199: 15-24.

11. Petridou E, Belechri M, Dessypris N, et al. Leptin and body mass index in relation to endometrial cancer risk. Ann Nutr Metab 2002; 46: 147-51.

12. Carino $C$, Olawaiye $A B$, Cherfils $S$, et al. Leptin regulation of proangiogenic molecules in benign and cancerous endometrial cells. Int J Cancer 2008; 123: 2782-90.

13. Tartaglia LA, Dembski $M$, Weng $X$, et al. Identification and expression cloning of a leptin receptor, OB-R. Cell 1995; 83: 1263-71.

14. Yuan SS, Tsai KB, Chung YF, et al. Aberrant expression and possible involvement of the leptin receptor in endometrial cancer. Gynecol Oncol 2004; 92: 769-75.

15. Bogusiewicz M, Semczuk A, Gogacz M, Skomra D, Jakowicki JA, Rechberger T. Lack of correlation between leptin receptor expression and PI3-K/Akt signaling pathway proteins immunostaining in endometrioid-type endometrial carcinomas. Cancer Lett 2006; 238: 61-8.

16. Jongen $\mathrm{V}$, Briet J, de Jong R, et al. Expression of estrogen receptor-alpha and -beta and progesterone receptor-A and -B in a large cohort of patients with endometrioid endometrial cancer. Gynecol Oncol 2009; 112: 537-42.

17. Kitawaki J, Koshiba H, Ishihara H, Kusuki I, Tsukamoto K, Honjo H. Expression of leptin receptor in human endometrium and fluctuation during the menstrual cycle. J Clin Endocrinol Metab 2000; 85: 1946-50.

18. Koda M, Sulkowska M, Wincewicz A, et al. Expression of leptin, leptin receptor, and hypoxia-inducible factor 1 alpha in human endometrial cancer. Ann N Y Acad Sci 2007; 1095: 90-8.

19. Méndez-López LF, Dávila-Rodríguez MI, Zavala-Pompa A, Torres-López E, González-Martínez BE, López-Cabanillas-Lomelí M. Expression of leptin receptor in endometrial biopsies of endometrial and ovarian cancer patients. Biomed Rep 2013; 1: 659-63.

20. Gates EJ, Hirschfield L, Matthews RP, Yap OW. Body mass index as a prognostic factor in endometrioid adenocar- 
cinoma of the endometrium. J Natl Med Assoc 2006; 98: 1814-22.

21. Silverberg SG. Problems in the differential diagnosis of endometrial hyperplasia and carcinoma. Mod Pathol 2000; 13: 309-27.

22. Noyes RW, Hertig AT, Rock J. Dating the endometrial biopsy. Am J Obstet Gynecol 1975; 122: 262-3.

23. Lacey JV, Jr Mutter GL, Nucci MR, et al. Risk of subsequent endometrial carcinoma associated with endometrial intraepithelial neoplasia classification of endometrial biopsies. Cancer 2008; 113: 2073-81.

24. Mazur MT, Kurman R. Diagnosis of endometrial biopsies and curettings: a practical approach (Second ed.). Springer Science and Business Media, New York 2005.

25. van Diest PJ, van Dam P, Henzen-Logmans SC, et al. A scoring system for immunohistochemical staining: consensus report of the task force for basic research of the EORTC-GCCG. European Organization for Research and Treatment of Cancer-Gynaecological Cancer Cooperative Group. J Clin Pathol 1997; 50: 801-4.

26. Pedrycz A, Siermontowski P. Influence of L-arginine on expression of HSP70 and p-53 proteins - early biomarkers of cellular danger in renal tubular cells. Immunohistochemical assessment. Arch Med Sci 2013; 9: 719-23.

27. Ruifrok AC, Johnston DA. Quantification of histochemical staining by color deconvolution. Anal Quant Cytol Histol 2001; 23: 291-9.

28. Cornish TC, Haluska MK. Color deconvolution for the analysis of tissue microarrays. Anal Quant Cytol Histo 2009; 31: 304-12.

29. Wells M. Hyperplasias of the endometrium. Elsevier Churchill Livingstone, Philadelphia 2004.

30. Gry M, Rimini R, Strömberg S, et al. Correlations between RNA and protein expression profiles in 23 human cell lines. BMC Genomics 2009; 10: 365.

31. Koshiba H, Kitawaki J, Ishihara $\mathrm{H}$, et al. Progesterone inhibition of functional leptin receptor mRNA expres sion in human endometrium. Mol Hum Reprod 2001; 7: $567-72$.

32. Henderson BE, Feigelson HS. Hormonal carcinogenesis. Carcinogenesis 2000; 21: 427-33.

33. Shimizu H, Shimomura $Y$, Nakanishi $Y$, et al. Estrogen increases in vivo leptin production in rats and human subjects. J Endocrinol 1997; 154: 285-92.

34. Duggal PS, Van Der Hoek KH, Milner CR, et al. The in vivo and in vitro effects of exogenous leptin on ovulation in the rat. Endocrinology 2000; 141: 1971-6.

35. Lacey JV Jr, Ioffe OB, Ronnett BM, et al. Endometrial carcinoma risk among women diagnosed with endometrial hyperplasia: the 34-year experience in a large health plan. Br J Cancer 2008; 98: 45-53.

36. Balmer NN, Richer JK, Spoelstra NS, Torkko KC, Lyle PL, Singh M. Steroid receptor coactivator AIB1 in endometrial carcinoma, hyperplasia and normal endometrium: correlation with clinicopathologic parameters and biomarkers. Mod Pathol 2006; 19: 1593-605.

37. Auwerx J, Staels B. Leptin. Lancet 1988; 351: 737-42.

38. Li M, Zhao L, Qi W, et al. Clinical implications and prognostic value of five biomarkers in endometrial carcinoma. Chin German J Clin Oncol 2013; 12: 586-91. 